\title{
Erratum to: Attention enhances apparent perceptual organization
}

\author{
Antoine Barbot $^{1,2}$ - Sirui Liu ${ }^{1}$ Ruth Kimchi ${ }^{3}$ - Marisa Carrasco ${ }^{1,4}$
}

Published online: 25 September 2017

(C) Psychonomic Society, Inc. 2017

\section{Erratum to: Psychon Bull Rev}

https://doi.org/10.3758/s13423-017-1365-x

This paper originally published with graphical errors in

Figures 1 and 4; it has been corrected.

The online version of the original article can be found at doi: https://doi. org/10.3758/s13423-017-1365-x

$\triangle$ Marisa Carrasco

marisa.carrasco@nyu.edu

1 Department of Psychology, New York University, New York, NY, USA

2 Center for Visual Science, University of Rochester, Rochester, NY, USA

3 Department of Psychology and Institute of Information Processing and Decision Making, University of Haifa, Haifa, Israel

4 Center of Neural Science, New York University, New York, NY, USA 